\title{
Determinants of Credit Rating Actions: Evidence from International Maritime Companies
}

\author{
Elif Korkmaz \\ Dept. of Business Administration, Faculty of Business, Dokuz Eylül University \\ Tinaztepe Campus, Buca, Izmir, Turkey \\ Tel: 90-232-453-5060Ｅ-mail: elif.korkmaz@deu.edu.tr \\ Ersin Firat Akgül \\ Dept. of Maritime Business Administration, Maritime Faculty \\ Bandırma Onyedi Eylül University, Central Campus, Bandırma, Balıkesir, Turkey \\ Tel: +90-266-717-0117_Ｅ-mail: akgulfirat@gmail.com \\ Seçil Sigalı \\ Dept. of Logistics Management, Maritime Faculty, Dokuz Eylül University \\ Tinaztepe Campus, Buca, Izmir, Turkey \\ Tel: 90-232-301-8801Ｅ-mail: secil.varan@deu.edu.tr
}

Received: Nov. 13, 2017 Accepted: Nov. 22, 2017 Published: Dec. 6, 2017

doi:10.5296/bmh.v5i2.12283～URL: http://dx.doi.org/10.5296/bmh.v5i2.12283

\begin{abstract}
This study aims to analyze the relevant factors in determining credit rating agency (CRA) rating actions for international maritime companies. The public disclosures regarding the credit rating actions within annual reports and the credit rating agencies' websites are analyzed by applying a content analysis for the period 2000-2017. The results of the content analysis indicate that the factor of "market conditions" has been disclosed as the main credit rating action determinant by the CRAs. This finding is in line with the argument that due to the high costs of obtaining the new and confidential information, CRAs tend to rely solely on
\end{abstract}




\section{Macrothink}

Business and Management Horizons

ISSN 2326-0297

2017, Vol. 5, No. 2

the market risk in most rating actions, rather than company-specific risk. Moreover, we determine that, after the 2008 financial crisis, CRA disclosures on company specific factors decrease dramatically. Furthermore, opacity prevails in observations regarding company-specific factors as "financial profile” and "corporate business profile”.

Keywords: Credit rating actions, Maritime companies, Public disclosures 


\section{Introduction}

The US subprime mortgage crisis in the mid-2008 has not only directly affected the financial industry in the US, but has also spread to other markets and countries due to the high proportion of CDOs securitized by mortgages (Stopford, 2013). With its effect felt more strongly in Europe, this crisis is seen as one of the worst financial crisis in history (Ackermann, 2008). A high balance of payments deficit within the US, the deflation of real estate assets, the liquidity excess due to the high supply of credits, and an artificial boom resulting in a supply increase within multiple sectors/countries- are the main factors that led to this crisis (De Monnie et al., 2009; Melvin \& Taylor, 2009; Lo Re, 2011). Following these developments, the maritime industry has not remained unaffected. The financial situation of the maritime industry has suffered from decreasing global demand and oversupply. This has in turn, led to new alliances, mergers, acquisitions and even failures as the Hanjin Shipping bankruptcy.

For the maritime companies, fleet growth and corresponding financial needs continue irrespective of the market conditions. Moreover, issues such as growing fleet age, higher insurance rates, increasing maintenance costs of the older vehicles, integration of technological developments, shifting quality standards, increasing competition and finally, increased demand (Brauner, 1994) continue to pressure the industry. Despite these, when seeking bank credit and corporate bond investors, maritime companies have faced more stringent financing conditions after 2008. One of which has been the application of strict financial covenants to risky industries (Mayer \& Brown, 2014). These developments act as motivation for this study, since higher credit ratings result in lower cost of capital (Kisgen \& Strahan, 2010).

Determination of the credit ratings require substantial CRA judgment as content such as firm's financial statements, management quality, and economic scenarios are employed under the rating process (Gonzales et al., 2004; Matthies, 2013). Due to the required judgment, credit rating agencies have been criticized for their failure to accurately predict credit risk. Bone \& Ribeiro (2009) and Elkhoury (2008) argues that the credit rating process lacks transparency, and the relevant factors taken into consideration by the credit agencies in determining the credit ratings can be considered as "opaque" information for the investors. Murcia et al. (2014) also argues that CRAs lack incentives to demand detailed information of debt issuers due to the high cost inherent in obtaining new information. CRAs tend to rely solely on market risk in most rating actions rather than company-specific risks, resulting in predictable credit ratings (Murcia et al., 2014).

This study analyzes the relevant factors in determining credit rating agencies' rating actions for international maritime companies. The sample of this study was collected among the firms listed under the "UNCTAD 2016 Top 20 Maritime Companies" that were rated by a CRA and were analyzed taking into consideration the Credit Risk Determinants of maritime companies.

The following section provides a brief literature review, while the third section presents the data and the methodology of the study. Next, the fourth section presents the findings and the 
final section concludes with suggestions for further research.

\section{Literature Review}

The credit risk measurement methods can be divided into two distinct categories; "traditional" and "modern". While expert systems, rating systems and credit scoring models can be categorized as traditional methods; an options-theoretic structural approach and a reduced form approach utilizing intensity-based models to estimate stochastic hazard rates can be considered as modern methods (Saunders et al., 2004).

Although the literature on credit risk measurement dates back to 1930s (Ramser \& Foster, 1931; Fitzpatrick, 1932; Winakor \& Smith, 1935), the pioneering study of Beaver's univariate analysis was conducted in 1966. Beaver (1966) documented that the failure of a firm can be correctly predicted to a much greater extent than would be expected from random prediction. Beaver's (1966) model was able to accurately classify $78 \%$ of the sample of firms at least five years before failure. However, Atiya (2001) argued that Beaver (1966)'s approach is inadequate for two main reasons. First, the analysis is based solely on a single financial ratio analysis at a time and second, a cutoff threshold is developed for each individual ratio. Later, Altman (1968) expanded the Beaver (1966) approach, and employed use of a multivariate discriminant analysis, which became a popular model extensively used in empirical research and in practice (Dichev, 1998).

After the 1990s, artificial neural networks have been employed to analyze the credit risk by multiple researchers (Odom \& Sharda, 1990; Wilson \& Sharda, 1994). Atiya (2001) explained the superiority of using non-linear approach instead of a linear approach by implying saturation effects and multiplicative factors in the relationships between the financial ratios and the prediction of default. Atiya (2001) also asserted that many commercial loan default prediction products (e.g., Moody's Public Firm Risk Model) and bankruptcy simulation models of leading global banks are based upon this approach.

The credit rating model is one of the commonly used models to measure credit risk. This model categorizes companies with regard to their credibility (Ong, 2003). The information provided by CRAs can be relevant for creditors and investors due to the following reasons;

i) Non-aligned credit rating agencies provide unbiased information;

ii) Employs experts in finance;

iii) Confidential information can be accessible for CRAs (Rajendran, 2015).

Rating agencies determine the credibility of companies by taking into consideration many factors, such as; macro and micro economic variables, financial strength, quality of management, and company competitiveness (Rajendran, 2015). Matthies (2013) provides an extensive review of the literature that analyzes the determinants of credit ratings and argues that the relevant factors of credit rating decisions are produced in three main categories. These are; financial ratios and financial data (e.g., firm specific factors such as leverage, liquidity, and firm size), corporate governance mechanisms (e.g. ownership structure, board independence) and macroeconomic factors (e.g., GDP) (Matthies 2013). 


\section{Data and Methodology}

This study investigates the credit rating actions produced by two competing CRAs, namely, Standard \& Poor's (S\&P) and Moody's. The AAA, AA, A, and BBB ratings are indicative of higher quality and are categorized as "investable", while indebtedness is rated as BB and lower. These ratings are considered as risky and "not investable” (Cantor \& Packer, 1994).

Moody's model for global maritime companies' credit risk measurement, aims to serve as a guideline for maritime industry stakeholders. The guidelines are disclosed to inform the stakeholders regarding the qualitative and quantitative factors that influence the companies' ratings (Moody's, 2014).

Moody's take into consideration the following five factors while assessing maritime company credit risk; 1. Scale, 2. Profitability, 3. Leverage and Coverage, 4. Fleet Characteristics, and 5. Financial Policy. These factors are followed by the sub-factors presented under Table 1.

Table 1. Relevant factors and sub-factors

\begin{tabular}{|l|l|l|l|}
\hline Broad Rating Factors & Factor Weighting & Rating Sub-Factor & Sub-Factor Weighting \\
\hline Scale & $20 \%$ & Revenue & $10 \%$ \\
\hline & & Size of Fleet & $10 \%$ \\
\hline Profitability & $17.5 \%$ & EBIT Margin & $10 \%$ \\
\hline Leverage and Coverage & $30 \%$ & ROA (NPATBUI/Total Assets) & $7.5 \%$ \\
\hline & & Debt / EBITDA & $10 \%$ \\
\hline & & RCF / Net Debt & $10 \%$ \\
\hline Fleet Characteristics & $17.5 \%$ & (FFO+Interest) / Interest Expense & $10 \%$ \\
\hline & & $\%$ Revenues from LT Charters & $7.5 \%$ \\
\hline Financial Policy & $15 \%$ & Unencumbered Assets & $10 \%$ \\
\hline Total & $100 \%$ & Financial Policy & $15 \%$ \\
\hline
\end{tabular}

Source: Moody’s Investor Service, Global Shipping Industry Methodologies Report (2014).

In addition to the above sector-specific factors, Moody's ratings also include the factors that are common across all industries, such as; ownership structure, liquidity, corporate legal structure, corporate governance and country specific risks (Moody's, 2014). Standard \& Poor's methodology of credit risk measurement involves the evaluation of business risk and financial risk, as shown in Figure 1. 


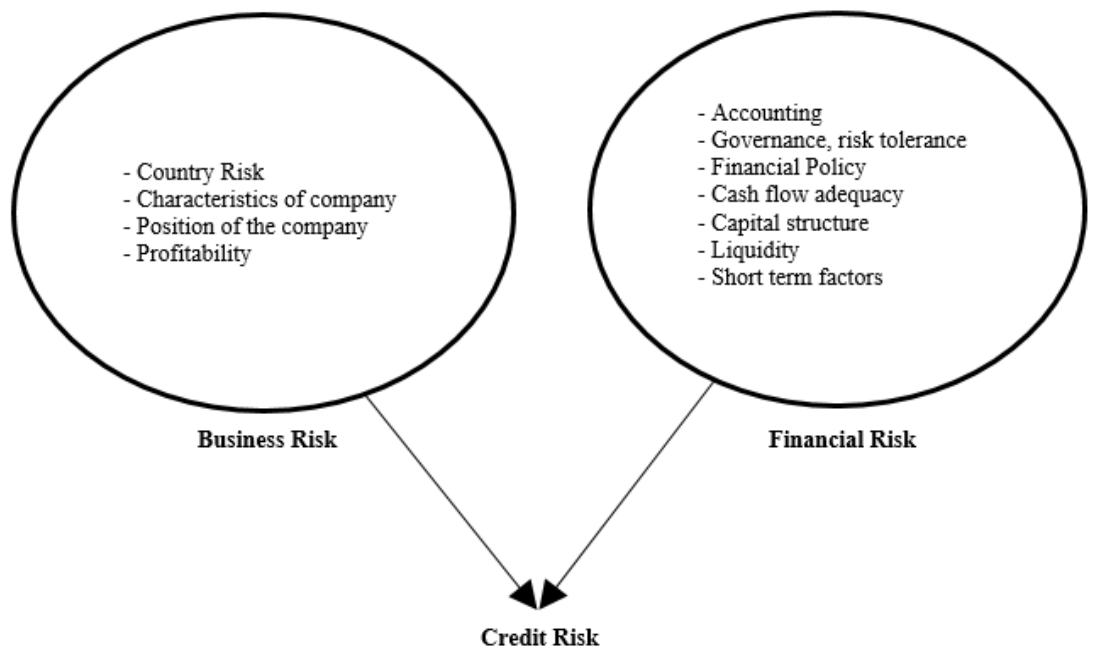

Figure 1. Credit rating factors

Source: S\&P Global Ratings

\subsection{Data and Analysis}

The sample was selected amongst the firms listed under the "UNCTAD 2016 the Global Top 20 Maritime Companies" as presented in Table 2 according to the end of 2015 figures. A content analysis is applied on the public disclosures regarding the credit actions of the sample companies. The public disclosures on the credit rating actions found under the annual reports of the companies and the credit rating agencies' websites are analyzed according to the factors that are presented in Matthies (2013) and the credit rating agencies' rating models. The period analyzed is 2000-2017 with 69 observations.

Table 2. Sample and sample data

\begin{tabular}{|l|l|l|l|l|l|l|}
\hline & Company & Headquarters & $\begin{array}{l}\text { TEU } \\
\text { Capacity }\end{array}$ & Revenue & $\begin{array}{l}\text { Founding } \\
\text { Year }\end{array}$ & $\begin{array}{l}\text { Number } \\
\text { of } \\
\text { Employee } \\
\text { s }\end{array}$ \\
\hline 1 & A.P. Moller Maersk & $\begin{array}{l}\text { Copenhagen, } \\
\text { Denmark }\end{array}$ & 3059984 & $\$ 40.3$ Billion (USD) & 1904 & 89.000 \\
\hline 2 & $\begin{array}{l}\text { Mediterranean } \\
\text { Shipping Company } \\
\text { S.A. (MSC) }\end{array}$ & $\begin{array}{l}\text { Geneva, } \\
\text { Switzerland }\end{array}$ & 2703404 & $\$ 28.2$ Billion (USD) & 1970 & 24.000 \\
\hline 3 & $\begin{array}{l}\text { CMA CGM Group } \\
4\end{array}$ & $\begin{array}{l}\text { Marseille, } \\
\text { France } \\
\text { (Group) COSCO) }\end{array}$ & 1873439 & $\$ 15.7$ Billion (USD) & 1978 & 22.000 \\
\hline 5 & Hapag-Lloyd & $\begin{array}{l}\text { Hamburg, } \\
\text { Germany }\end{array}$ & 978663 & $\$ 9.2$ billion (USD) & 1847 & 130.000 \\
\hline 6 & Evergreen Marine & $\begin{array}{l}\text { Taoyuan City, } \\
\text { Taiwan }\end{array}$ & 949492 & $\$ 4.6$ billion (USD) & 1968 & 3.389 \\
\hline
\end{tabular}




\section{Ml Macrothink}

Business and Management Horizons

ISSN 2326-0297

2017, Vol. 5, No. 2

\begin{tabular}{|c|c|c|c|c|c|c|}
\hline 7 & Hamburg Süd Group & $\begin{array}{l}\text { Hamburg, } \\
\text { Germany }\end{array}$ & 670029 & $\$ 6.9$ billion (USD) & 1871 & 5.360 \\
\hline 8 & Hanjin Shipping & $\begin{array}{ll}\text { Seoul, } & \text { South } \\
\text { Korea } & \\
\end{array}$ & 648043 & $\$ 8.3$ billion (USD) & 1977 & 5.800 \\
\hline 9 & $\begin{array}{lr}\text { Orient } & \text { Overseas } \\
\text { Container } & \text { Line } \\
\text { (OOCL) } & \\
\end{array}$ & $\begin{array}{l}\text { Hong Kong, } \\
\text { China }\end{array}$ & 571429 & \$ 5.9 billion (USD) & 1969 & 9889 \\
\hline 10 & $\begin{array}{l}\text { Neptune Orient Lines } \\
\text { - American President } \\
\text { Lines }\end{array}$ & $\begin{array}{l}\text { Marseille, } \\
\text { France }\end{array}$ & 567635 & $\$ 6.02$ billion (USD) & 1978 & over 7000 \\
\hline 11 & $\begin{array}{l}\text { Mitsui Osaka Shosen } \\
\text { Kaisha Lines }\end{array}$ & Tokyo, Japan & 542909 & \$ 15.3 million (USD) & 1878 & $\begin{array}{l}966 \\
\text { (Number } \\
\text { of MOL } \\
\text { Group } \\
\text { employees: } \\
\text { 10,794) }\end{array}$ \\
\hline 12 & $\begin{array}{l}\text { Yang Ming Marine } \\
\text { Transport }\end{array}$ & $\begin{array}{l}\text { Keelung, } \\
\text { Taiwan }\end{array}$ & 542127 & $\$ 4.2$ million (USD) & 1972 & N/A \\
\hline 13 & $\begin{array}{l}\text { United Arab Shipping } \\
\text { Company }\end{array}$ & $\begin{array}{l}\text { Dubai, United } \\
\text { Arab Emirates }\end{array}$ & 452510 & N/A & 1976 & N/A \\
\hline 14 & Nippon Yusen Kaisha & Tokyo, Japan & 493443 & \$ 20 million (USD) & 1885 & 35.935 \\
\hline 15 & $\begin{array}{l}\text { Hyundai Merchant } \\
\text { Marine }\end{array}$ & $\begin{array}{l}\text { Seoul, South } \\
\text { Korea }\end{array}$ & 381728 & \$ 5.1 million (USD) & 1979 & N/A \\
\hline 16 & 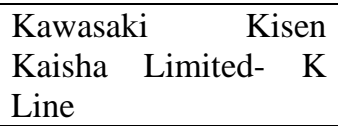 & Tokyo, Japan & 397557 & \$ 11 million (USD) & 1919 & 735 \\
\hline 17 & $\begin{array}{ll}\text { ZIM } & \text { Integrated } \\
\text { Shipping Services }\end{array}$ & Haifa, Israel & 368884 & \$ 2,9 million (USD) & 1945 & N/A \\
\hline 18 & $\begin{array}{ll}\text { Pacific } & \text { International } \\
\text { Lines } & \\
\end{array}$ & Singapore & 336699 & $\begin{array}{ll}\text { about } & \$ 3,000.000 \\
\text { (USD) } & \\
\end{array}$ & 1967 & 18000 \\
\hline 19 & Wan Hai Lines & Taiwan & 217847 & 63.86 billion TWD & 1965 & 3162 \\
\hline 20 & X-Press Feeders & Singapore & 116709 & N/A & 1972 & 260 \\
\hline
\end{tabular}

\section{Findings}

As shown in Table 3 and Figure 2, it is determined that the factor of "market conditions" (as macro-economic factor) has been disclosed as the main credit rating action determinant by the CRAs under the 69 observations. This finding is in line with Murcia et al. (2014)'s argument that due to the high costs of obtaining the new and confidential information, credit rating agencies tend to rely solely on the market risk in most rating actions rather than company-specific risk, thus produce predictable credit ratings (Murcia et. al. 2014).

The findings are presented in Table 3 in detail. 
Table 3. The Findings

\begin{tabular}{|c|c|c|c|c|c|c|c|}
\hline $\begin{array}{l}\text { Company } \\
\text { Name } \\
\end{array}$ & Date & $\begin{array}{l}\text { Rating } \\
\text { Agency }\end{array}$ & $\begin{array}{l}\text { Rating } \\
\text { Action } \\
\end{array}$ & Rationale & Outlook & Further Expectations & $\begin{array}{l}\text { Relevant } \\
\text { Factors } \\
\end{array}$ \\
\hline $\begin{array}{l}\text { Nippon } \\
\text { Yusen } \\
\text { Kaisha }\end{array}$ & $\begin{array}{l}1.03 . \\
00\end{array}$ & Moody's & Baa2 & $\begin{array}{l}\text { •Company’s and its } \\
\text { subsidiaries' } \\
\text { characteristics }\end{array}$ & Positive & $\begin{array}{l}\text { •NYK's improving } \\
\text { performance will continue } \\
\text { and the company's financial } \\
\text { fundamentals will be } \\
\text { strengthened in the near to } \\
\text { medium term }\end{array}$ & $\begin{array}{l}\text { Corporate } \\
\text { Business } \\
\text { Profile }\end{array}$ \\
\hline $\begin{array}{l}\text { Mitsui } \\
\text { O.S.K } \\
\text { Lines }\end{array}$ & $\begin{array}{l}2.03 . \\
00\end{array}$ & Moody's & Ba1 & $\begin{array}{l}\text {-Improvement } \\
\text { operations caused by } \\
\text { rising freight rates }\end{array}$ & Positive & $\begin{array}{l}\text {-Expectation of MOL's } \\
\text { improving performance will } \\
\text { continue to contribute to its } \\
\text { earnings. }\end{array}$ & $\begin{array}{l}\text { Market } \\
\text { Conditions }\end{array}$ \\
\hline $\begin{array}{l}\text { Nippon } \\
\text { Yusen } \\
\text { Kaisha }\end{array}$ & $\begin{array}{l}26.12 \\
.01\end{array}$ & Moody’s & Baa2 & $\begin{array}{l}\text {-Good operating } \\
\text { performance due to the } \\
\text { industry upcycle and } \\
\text { robust world trade } \\
\text { volume resulting from } \\
\text { the generally strong } \\
\text { global economy }\end{array}$ & Stable & $\begin{array}{l}\text { - The challenging market } \\
\text { conditions } \\
\text { deteriorating conditions } \\
\text { evident in } 2001 \text { represent a } \\
\text { broader industry down } \\
\text { cycle }\end{array}$ & $\begin{array}{l}\text { Market } \\
\text { Conditions }\end{array}$ \\
\hline $\begin{array}{l}\text { Mitsui } \\
\text { O.S.K } \\
\text { Lines }\end{array}$ & $\begin{array}{l}26.12 \\
.01\end{array}$ & Moody’s & Ba1 & $\begin{array}{l}\text {-Deteriorating market } \\
\text { conditions }\end{array}$ & Stable & $\begin{array}{l}\text { - It is expected that the } \\
\text { deteriorating conditions } \\
\text { evident in } 2001 \text { represent a } \\
\text { broader industry down } \\
\text { cycle }\end{array}$ & $\begin{array}{l}\text { Market } \\
\text { Conditions }\end{array}$ \\
\hline $\begin{array}{l}\text { Nippon } \\
\text { Yusen } \\
\text { Kaisha }\end{array}$ & $\begin{array}{l}8.01 . \\
02\end{array}$ & Moody’s & Baa2 & $\begin{array}{l}\text { Highly leveraged } \\
\text { financial position and } \\
\text { high fixed cost structure } \\
\text { requiring a large } \\
\text { amount of investment to } \\
\text { remain competitive }\end{array}$ & Stable & $\begin{array}{l}\text {-The deteriorating } \\
\text { conditions evident in } 2001 \\
\text { represent a broader industry } \\
\text { down cycle }\end{array}$ & Leverage \\
\hline $\begin{array}{l}\text { CMA } \\
\text { CGM }\end{array}$ & $\begin{array}{l}22.04 \\
.03\end{array}$ & Moody’s & $\mathrm{Ba} 2$ & $\begin{array}{l}\text {-CMA CGM's high } \\
\text { adjusted debt leverage } \\
\text { •CMA CGM's high } \\
\text { adjusted debt leverage } \\
\text { and expected } 2003 \\
\text { negative free cash flow } \\
\text {-Fluctuations in fuel } \\
\text { prices }\end{array}$ & Stable & $\begin{array}{l}\text { - Expected that } 2003 \\
\text { negative free cash flow } \\
\text {-Moody's expectation of } \\
\text { continual growth in the } \\
\text { container industry, gradual } \\
\text { firming of freight rates over } \\
\text { the short term and an } \\
\text { expectation of lower annual } \\
\text { ship supply during } 2003 \\
\text { and } 2004 \text { which should } \\
\text { stabilize the overall } \\
\text { dynamics of the industry }\end{array}$ & Leverage \\
\hline $\begin{array}{l}\text { Nippon } \\
\text { Yusen } \\
\text { Kaisha }\end{array}$ & $\begin{array}{l}4.08 . \\
04\end{array}$ & Moody's & Review & $\begin{array}{l}\text {-NYK is likely to } \\
\text { reinforce its market } \\
\text { position by improving } \\
\text { its operating } \\
\text { performance and } \\
\text { earning stability over } \\
\text { the medium term }\end{array}$ & Stable & •Possible upgrade & $\begin{array}{l}\text { Operating } \\
\text { Performanc } \\
\text { e }\end{array}$ \\
\hline $\begin{array}{l}\text { Mitsui } \\
\text { O.S.K } \\
\text { Lines }\end{array}$ & $\begin{array}{l}4.08 \\
04\end{array}$ & Moody’s & Review & $\begin{array}{l}\text {-Stability of MOL's } \\
\text { operating performance } \\
\text { - Cash-generating } \\
\text { ability is likely to } \\
\text { increase }\end{array}$ & Stable & •Possible upgrade & $\begin{array}{l}\text { Operating } \\
\text { Performanc } \\
\text { e }\end{array}$ \\
\hline $\begin{array}{l}\text { Nippon } \\
\text { Yusen }\end{array}$ & $\begin{array}{l}15.11 \\
.04\end{array}$ & Moody’s & A3 & $\begin{array}{l}\text { •Significant } \\
\text { improvement in NYK's }\end{array}$ & Stable & N/A & $\begin{array}{l}\text { Cash flow } \\
\text { generation }\end{array}$ \\
\hline
\end{tabular}




\section{Macrothink}

Business and Management Horizons

ISSN 2326-0297

2017, Vol. 5, No. 2

\begin{tabular}{|c|c|c|c|c|c|c|c|}
\hline Kaisha & & & & $\begin{array}{l}\text { financial position } \\
\text { - Cash flow generation } \\
\text { in recent years }\end{array}$ & & & \\
\hline $\begin{array}{l}\text { Mitsui } \\
\text { O.S.K } \\
\text { Lines }\end{array}$ & $\begin{array}{l}15.11 \\
.04\end{array}$ & Moody's & Baa2 & $\begin{array}{l}\text { - Reduction of the cost } \\
\text { and improving the } \\
\text { efficiency of its } \\
\text { shipping operations and } \\
\text { enhanced their global } \\
\text { competitiveness } \\
\text { - Investments to enhance } \\
\text { the quality of its fleet }\end{array}$ & Stable & $\begin{array}{l}\text {-Moody's believes the } \\
\text { sustainable cash flow will } \\
\text { continue to improve its } \\
\text { capital structure going } \\
\text { forward } \\
\text { - Rapid growth of Asian } \\
\text { demand and rising fuel } \\
\text { prices may raise the } \\
\text { volatility of freight prices in } \\
\text { the future }\end{array}$ & $\begin{array}{l}\text { Operating } \\
\text { Performanc } \\
\text { e }\end{array}$ \\
\hline $\begin{array}{l}\text { Mitsui } \\
\text { O.S.K } \\
\text { Lines }\end{array}$ & $\begin{array}{l}25.03 \\
.05\end{array}$ & Moody's & Baa2 & $\begin{array}{l}\text { •Improving financial } \\
\text { profile }\end{array}$ & Stable & N/A & $\begin{array}{l}\text { Financial } \\
\text { profile }\end{array}$ \\
\hline $\begin{array}{l}\text { Mitsui } \\
\text { O.S.K } \\
\text { Lines }\end{array}$ & $\begin{array}{l}12.08 \\
.05\end{array}$ & Moody's & Baa2 & $\begin{array}{l}\text {-MOL is likely to } \\
\text { strengthen its financial } \\
\text { flexibility despite its } \\
\text { intensive capital } \\
\text { expenditure plan for } \\
\text { fleet expansion } \\
\text {-Greatly increase } \\
\text { operating profit }\end{array}$ & Positive & $\begin{array}{l}\text {-Moody's believes the cash } \\
\text { flow generated from its } \\
\text { highly competitive fleet and } \\
\text { diversified business } \\
\text { operations is likely to } \\
\text { improve its capital structure } \\
\text { going forward }\end{array}$ & Growth \\
\hline $\begin{array}{l}\text { Mitsui } \\
\text { O.S.K } \\
\text { Lines }\end{array}$ & $\begin{array}{l}13.10 \\
.05\end{array}$ & Moody's & Review & $\begin{array}{l}\cdot \text { MOL nearly doubled } \\
\text { its operating profit to } \\
\text { JPY } 171.8 \text { billion for } \\
\text { fiscal 2004 (ended } \\
\text { March 2005) from JPY } \\
92.1 \text { billion for fiscal } \\
2003\end{array}$ & Positive & •Possible upgrade & $\begin{array}{l}\text { Profitabilit } \\
\text { y }\end{array}$ \\
\hline $\begin{array}{l}\text { CMA } \\
\text { CGM }\end{array}$ & $\begin{array}{l}1.11 . \\
05\end{array}$ & Moody's & $\mathrm{Ba} 2$ & $\begin{array}{l}\text {-CMA CGM's ongoing } \\
\text { positive operational and } \\
\text { financial performance } \\
\text { over the last several } \\
\text { years } \\
\text { - CMA CGM has one of } \\
\text { the youngest fleet in the } \\
\text { industry }\end{array}$ & Positive & $\begin{array}{l}\text { - Demand increase on these } \\
\text { routes which is expected to } \\
\text { continue well into } 2006 \\
\text {-Increase the supply in } \\
\text { shipping industry next } 3 \\
\text { years with deliveries of new } \\
\text { big ships }\end{array}$ & $\begin{array}{l}\text { Operating } \\
\text { Performanc } \\
\text { e }\end{array}$ \\
\hline $\begin{array}{l}\text { Mitsui } \\
\text { O.S.K } \\
\text { Lines }\end{array}$ & $\begin{array}{l}24.11 \\
.05\end{array}$ & Moody's & Baa1 & $\begin{array}{l}\text {-Increase operating } \\
\text { profit in the fiscal } \\
\text { half-year ending despite } \\
\text { recent fuel price rises } \\
\text { - Reduction of costs due } \\
\text { to reducing personnel } \\
\text { expenses }\end{array}$ & Stable & $\begin{array}{l}\text {-Moody's believes that } \\
\text { MOL's improvements in } \\
\text { both profitability and } \\
\text { stability of cash flow } \\
\text { generation will contribute } \\
\text { to stabilizing its financial } \\
\text { structure, even if market } \\
\text { conditions deteriorate in the } \\
\text { future }\end{array}$ & $\begin{array}{l}\text { Profitabilit } \\
\text { y }\end{array}$ \\
\hline $\begin{array}{l}\text { CMA } \\
\text { CGM }\end{array}$ & $\begin{array}{l}11.07 \\
.06\end{array}$ & Moody's & Ba1 & $\begin{array}{lr}\text { •CMA } & \text { CGM's } \\
\text { strengthening } & \text { market } \\
\text { position in } & \text { Ocean } \\
\text { Containers } & \text { Shipping } \\
\text { industry } & \\
\end{array}$ & Stable & N/A & Size \\
\hline $\begin{array}{l}\text { Mitsui } \\
\text { O.S.K } \\
\text { Lines }\end{array}$ & $\begin{array}{l}15.02 \\
.07\end{array}$ & Moody's & Baa1 & $\begin{array}{l}\text { - Reduction of debt level } \\
\text { - Fleet expansion } \\
\text { - Long-term } \\
\text { transportation contracts } \\
\text { with customers } \\
\end{array}$ & Positive & $\begin{array}{l}\text {-Expectation of high level } \\
\text { of operating profit }\end{array}$ & Leverage \\
\hline
\end{tabular}




\section{Macrothink Mnstitute}

\begin{tabular}{|c|c|c|c|c|c|c|c|}
\hline $\begin{array}{l}\text { Mitsui } \\
\text { O.S.K } \\
\text { Lines }\end{array}$ & $\begin{array}{l}19.10 \\
.07\end{array}$ & Moody's & Review & $\begin{array}{l}\cdot \mathrm{MOL} \text { is likely to } \\
\text { further strengthen its } \\
\text { financial profile over } \\
\text { the medium term, } \\
\text { despite its intensive } \\
\text { capital expenditure to } \\
\text { expand the fleet }\end{array}$ & Positive & •Possible upgrade & $\begin{array}{l}\text { Financial } \\
\text { profile }\end{array}$ \\
\hline $\begin{array}{l}\text { Mitsui } \\
\text { O.S.K } \\
\text { Lines }\end{array}$ & $\begin{array}{l}29.11 \\
.07\end{array}$ & Moody’s & A3 & $\begin{array}{l}\text {-Improving its } \\
\text { profitability by reducing } \\
\text { various fixed costs and } \\
\text { improving the } \\
\text { efficiency of its } \\
\text { shipping operations }\end{array}$ & Stable & $\begin{array}{l}\text {-Moody's believes that } \\
\text { MOL will achieve stronger } \\
\text { financial flexibility, even if } \\
\text { market conditions } \\
\text { deteriorate in the future }\end{array}$ & $\begin{array}{l}\text { Profitabilit } \\
\mathrm{y}\end{array}$ \\
\hline $\begin{array}{ll}\text { Wan } & \text { Hai } \\
\text { Lines } & \end{array}$ & $\begin{array}{l}4.12 . \\
08\end{array}$ & $\mathrm{~S} \& \mathrm{P}$ & $\mathrm{BB}+$ & N/A & Negative & N/A & N/A \\
\hline $\begin{array}{l}\text { CMA } \\
\text { CGM }\end{array}$ & $\begin{array}{l}16.12 \\
.08\end{array}$ & Moody’s & Ba1 & $\begin{array}{l}\text {-The change of outlook } \\
\text { to negative reflects } \\
\text { Moody's increasing } \\
\text { concerns over the } \\
\text { uncertainty. The current } \\
\text { challenging } \\
\text { environment will } \\
\text { continue to exert } \\
\text { pressure on CMA } \\
\text { CGM's credit profile } \\
\text {-Difficult market } \\
\text { conditions but strong } \\
\text { positions of CMA CGM }\end{array}$ & Negative & $\begin{array}{l}\text { - Inceasing pressure on the } \\
\text { container shipping market } \\
\text { - Higher operating costs } \\
\text { from bunker and chartering } \\
\text { costs } \\
\text {-CMA CGM will have to } \\
\text { finance its heavy capex } \\
\text { program in a context of } \\
\text { slower growth prospects } \\
\text { and potential over-capacity } \\
\text { in the container shipping } \\
\text { market }\end{array}$ & $\begin{array}{l}\text { Market } \\
\text { Conditions }\end{array}$ \\
\hline $\begin{array}{l}\text { Yang Ming } \\
\text { Marine } \\
\text { Transport } \\
\text { Co. }\end{array}$ & $\begin{array}{l}26.05 \\
.09\end{array}$ & $\begin{array}{l}\text { S\&P } \\
\text { (Accordi } \\
\text { ng to } \\
\text { Taiwan } \\
\text { national } \\
\text { Scale) }\end{array}$ & A-3 & N/A & N/A & N/A & N/A \\
\hline $\begin{array}{l}\text { CMA } \\
\text { CGM }\end{array}$ & $\begin{array}{l}5.06 . \\
09\end{array}$ & Moody’s & B1 & $\begin{array}{lr}\text {-Global } & \text { economic } \\
\text { downturn } & \\
\text { •Weak } & \text { market } \\
\text { conditions r } & \\
\text {-Cancellation r or } \\
\text { postponing deliveries of } \\
\text { new ships }\end{array}$ & Negative & $\begin{array}{l}\text { - Negative Cash flows } \\
\text { - Lenders are likely to } \\
\text { remain supportive and that } \\
\text { the liquidity situation, } \\
\text { which partly relies on asset } \\
\text { sales, will remain adequate }\end{array}$ & $\begin{array}{l}\text { Market } \\
\text { Conditions }\end{array}$ \\
\hline $\begin{array}{l}\text { Nippon } \\
\text { Yusen } \\
\text { Kaisha }\end{array}$ & $\begin{array}{l}15.06 \\
.09\end{array}$ & Moody’s & A3 & $\begin{array}{l}\text {-NYK's financial } \\
\text { leverage is likely to } \\
\text { remain high comparing } \\
\text { with shipping industry } \\
\text {-NYK’s static position } \\
\text { in maritime sector }\end{array}$ & Negative & $\begin{array}{l}\text {-Projected lower operating } \\
\text { profit comparing with } \\
\text { previous year }\end{array}$ & Leverage \\
\hline $\begin{array}{l}\text { Nippon } \\
\text { Yusen } \\
\text { Kaisha }\end{array}$ & $\begin{array}{l}29.10 \\
.09\end{array}$ & Moody’s & A3 & $\begin{array}{l}\cdot \text { Market conditions } \\
\text {-Continuous pressure } \\
\text { on the earnings }\end{array}$ & Negative & •Possible downgrade & $\begin{array}{l}\text { Market } \\
\text { Conditions }\end{array}$ \\
\hline $\begin{array}{l}\text { Mitsui } \\
\text { O.S.K } \\
\text { Lines }\end{array}$ & $\begin{array}{l}29.10 \\
.09\end{array}$ & Moody’s & A3 & $\begin{array}{l}\text {-Market conditions } \\
\text {-Downward revised } \\
\text { operating profit } \\
\text { expectations of the } \\
\text { company } \\
\text { •Efforts on increasing } \\
\text { freight rates by reducing } \\
\text { capacity through laying } \\
\text { up and selling ships }\end{array}$ & Negative & $\begin{array}{l}\text { •Expectation of slower } \\
\text { earnings recovery }\end{array}$ & $\begin{array}{l}\text { Market } \\
\text { Conditions }\end{array}$ \\
\hline
\end{tabular}




\section{Macrothink Mnstitute}

\begin{tabular}{|c|c|c|c|c|c|c|c|}
\hline $\begin{array}{l}\text { Nippon } \\
\text { Yusen } \\
\text { Kaisha }\end{array}$ & $\begin{array}{l}9.2 .1 \\
0\end{array}$ & Moody’s & Baa1 & $\begin{array}{l}\text { •High debt/EBITDA } \\
\text { ratio }\end{array}$ & Negative & $\begin{array}{l}\text {-NYK will be unable to } \\
\text { recover levels of } \\
\text { profitability and financial } \\
\text { leverage consistent with its } \\
\text { previous rating until at least } \\
\text { March, } 2013\end{array}$ & Leverage \\
\hline $\begin{array}{l}\text { Pacific } \\
\text { Internation } \\
\text { al Line }\end{array}$ & $\begin{array}{l}30.04 \\
.10\end{array}$ & Moody’s & B1 & $\begin{array}{l}\text { - Long track record of } \\
\text { operating through } \\
\text { various cycles in the } \\
\text { liner industry } \\
\text {-PIL's competitive } \\
\text { profile and strong niche } \\
\text { market position in the } \\
\text { fast growing economies } \\
\text { China, Middle East and } \\
\text { Africa } \\
\text { - Young and flexible } \\
\text { fleet } \\
\text { - Operating strategy and } \\
\text { good liquidity } \\
\text { management }\end{array}$ & Stable & $\begin{array}{l}\text {-PIL will gradually improve } \\
\text { its operating performance } \\
\text {-PIL's liquidity is sufficient } \\
\text { to see it through the next } \\
\text { two years, and to take } \\
\text { delivery of its new vessels } \\
\text { without incurring additional } \\
\text { debt }\end{array}$ & $\begin{array}{l}\text { Operating } \\
\text { Performanc } \\
\text { e }\end{array}$ \\
\hline $\begin{array}{l}\text { Hapag } \\
\text { Lloyd }\end{array}$ & $\begin{array}{l}23.09 \\
.10\end{array}$ & Moody’s & $\begin{array}{l}\text { B1 } \\
\text { (CFR) } \\
\text { B3 } \\
\text { (PDR) }\end{array}$ & $\begin{array}{l}\text {-The credit rating action } \\
\text { was based on limited } \\
\text { historical data and } \\
\text { characteristics of Hapag } \\
\text { Lloyd. }\end{array}$ & Stable & $\begin{array}{l}\text {-Extremely weak financial } \\
\text { results and credit metrics } \\
\text { that HL will improve going } \\
\text { forward }\end{array}$ & $\begin{array}{l}\text { Financial } \\
\text { profile }\end{array}$ \\
\hline $\begin{array}{l}\text { CMA } \\
\text { CGM }\end{array}$ & $\begin{array}{l}6.04 \\
11\end{array}$ & Moody’s & Ba3 & $\begin{array}{l}\text {-Weakness of CMA } \\
\text { CGM's credit metrics } \\
\text { despite its strong } \\
\text { business profile } \\
\text {-Improving financial } \\
\text { profile } \\
\text {-Strong business profile } \\
\text { due to its leading } \\
\text { market positions gained } \\
\text { from the successful } \\
\text { commercial rand } \\
\text { operational strategies } \\
\text { implemented r by } \\
\text { management and good } \\
\text { cash flow generation } \\
\text {-Company's strong } \\
\text { asset base r }\end{array}$ & Stable & $\begin{array}{l}\text { - Credit metrics will remain } \\
\text { weak in the near future } \\
\text {-After the market recovery, } \\
\text { the agreement signed with } \\
\text { Yildirim Holding and the } \\
\text { signing of the restructuring } \\
\text { agreements with the banks, } \\
\text { CMA CGM will have } \\
\text { stabilized its capital } \\
\text { structure and liquidity } \\
\text { profile }\end{array}$ & $\begin{array}{l}\text { Financial } \\
\text { profile }\end{array}$ \\
\hline $\begin{array}{l}\text { CMA } \\
\text { CGM }\end{array}$ & $\begin{array}{l}8.09 \\
11\end{array}$ & Moody’s & B1 & $\begin{array}{l}\cdot \text { Difficult operating } \\
\text { environment with a } \\
\text { financial performance } \\
\text { materially below } \\
\text { Moody's previous } \\
\text { expectations despite a } \\
\text { growth of volumes and } \\
\text { revenues year on year } \\
\end{array}$ & Negative & $\begin{array}{l}\text {-Iincreased capacity will } \\
\text { cause highly competition }\end{array}$ & $\begin{array}{l}\text { Market } \\
\text { Conditions }\end{array}$ \\
\hline $\begin{array}{l}\text { Yang Ming } \\
\text { Marine } \\
\text { Transport } \\
\text { Co. }\end{array}$ & $\begin{array}{l}11.10 \\
.11\end{array}$ & $\begin{array}{l}\text { S\&P } \\
\text { (Accordi } \\
\text { ng to } \\
\text { Taiwan } \\
\text { national } \\
\text { Scale) }\end{array}$ & BBB & N/A & Negative & N/A & N/A \\
\hline $\begin{array}{l}\text { CMA } \\
\text { CGM }\end{array}$ & $\begin{array}{l}2.12 . \\
11\end{array}$ & Moody's & B2 & $\begin{array}{l}\text {-CMA CGM's weak } \\
\text { performance for the }\end{array}$ & Negative & $\begin{array}{l}\text {-Industry conditions would } \\
\text { not further worsen and that }\end{array}$ & $\begin{array}{l}\text { Market } \\
\text { Conditions }\end{array}$ \\
\hline
\end{tabular}




\begin{tabular}{|c|c|c|c|c|c|c|c|}
\hline & & & & $\begin{array}{l}\text { third quarter } \\
\text {-Poor performance of } \\
\text { the industry during the } \\
\text { peak season caused by } \\
\text { oversupply }\end{array}$ & & $\begin{array}{l}\text { actually freight rates } \\
\text { recover, at least modestly, } \\
\text { in the last weeks of the year } \\
\text { as well as in 2012, } \\
\text { following the withdrawal of } \\
\text { capacity currently } \\
\text { underway on the main trade } \\
\text { line } \\
\text {-Moody's assumption that } \\
\text { CMA CGM's lenders will } \\
\text { continue to remain } \\
\text { supportive of CMA CGM }\end{array}$ & \\
\hline $\begin{array}{l}\text { Nippon } \\
\text { Yusen } \\
\text { Kaisha }\end{array}$ & $\begin{array}{l}2.02 . \\
12\end{array}$ & Moody's & Baа2 & $\begin{array}{l}\text {-Weak cash flow } \\
\text { generating ability due to } \\
\text { bad conditions of } \\
\text { maritime industry } \\
\text { (oversupply of vessels } \\
\text { and downward pressure } \\
\text { on freight rates) } \\
\text { •Higher leverage } \\
\text {-NYK maintains profits } \\
\text { and strong customer } \\
\text { relationships, based on } \\
\text { its relatively large } \\
\text { long-term contract } \\
\text { businesses and stable } \\
\text { non-shipping operations }\end{array}$ & Negative & $\begin{array}{l}\text {-Currently, Japanese } \\
\text { companies enjoy access to } \\
\text { the banking system which is } \\
\text { liquid and willing to lend. } \\
\text { This environment is } \\
\text { expected to continue and to } \\
\text { provide NYK with the } \\
\text { capability to withstand the } \\
\text { financial stress that is } \\
\text { expected to continue for the } \\
\text { intermediate term }\end{array}$ & $\begin{array}{l}\text { Market } \\
\text { Conditions }\end{array}$ \\
\hline $\begin{array}{l}\text { Mitsui } \\
\text { O.S.K } \\
\text { Lines }\end{array}$ & $\begin{array}{l}2.02 . \\
12\end{array}$ & Moody's & Baa1 & $\begin{array}{l}\text { - Weak cash flow } \\
\text { - Challenging market } \\
\text { conditions due to } \\
\text { oversupply of vessels } \\
\text {-Maintaining profits } \\
\text { and strong customer } \\
\text { relationship } \\
\text {-MOL's stable and } \\
\text { strong relationships } \\
\text { with its major banks }\end{array}$ & Negative & $\begin{array}{l}\text { - Weak financial metrics } \\
\text { - Moody's believes that the } \\
\text { volatility of the industry has } \\
\text { been increasing, while its } \\
\text { overall tolerance for } \\
\text { business risk is weakening } \\
\text {-Adverse operating } \\
\text { environment will continue } \\
\text { due to demand-supply gaps } \\
\text { and the uncertainty over the } \\
\text { global economy }\end{array}$ & $\begin{array}{l}\text { Market } \\
\text { Conditions }\end{array}$ \\
\hline $\begin{array}{l}\text { CMA } \\
\text { CGM }\end{array}$ & $\begin{array}{l}13.03 \\
.12\end{array}$ & Moody's & B3 & $\begin{array}{l}\text { - Weaker financial } \\
\text { results than } \\
\text { expectations cause } \\
\text { liquidity negatively } \\
\text { •Poor performance of } \\
\text { the container shipping } \\
\text { industry during 2011 } \\
\text { caused by oversupply } \\
\text { •Higher bunker costs } \\
\text { but lower freight rates }\end{array}$ & N/A & $\begin{array}{l}\text { - As a result of the reduction } \\
\text { of capacity on the main } \\
\text { trade lines that is currently } \\
\text { under way, freight rates } \\
\text { could recover over the } \\
\text { coming months as the } \\
\text { volume of traffic increases }\end{array}$ & $\begin{array}{l}\text { Market } \\
\text { Conditions }\end{array}$ \\
\hline $\begin{array}{l}\text { CMA } \\
\text { CGM }\end{array}$ & $\begin{array}{l}21.03 \\
.12\end{array}$ & Moody’s & B3 & $\begin{array}{l}\text {-Review of the previous } \\
\text { rating action }\end{array}$ & Positive & $\begin{array}{l}\text { - Sustained } \\
\text { performance in } 2013 \\
\text { leading to steadily } \\
\text { improving credit metrics } \\
\text { over time, provided that } \\
\text { there are no significant } \\
\text { macro-shocks that would } \\
\text { affect the container } \\
\text { shipping market }\end{array}$ & $\begin{array}{l}\text { Operating } \\
\text { Performanc } \\
\text { e }\end{array}$ \\
\hline $\begin{array}{l}\text { Wan Hai } \\
\text { Lines }\end{array}$ & $\begin{array}{l}26.04 \\
.12 \\
\end{array}$ & $\begin{array}{l}\text { S\&P } \\
\text { (Accordi }\end{array}$ & BBB & N/A & Negative & N/A & N/A \\
\hline
\end{tabular}




\begin{tabular}{|c|c|c|c|c|c|c|c|}
\hline & & $\begin{array}{l}\text { ng to } \\
\text { China } \\
\text { national } \\
\text { Scale) }\end{array}$ & & & & & \\
\hline $\begin{array}{l}\text { Wan Hai } \\
\text { Lines }\end{array}$ & $\begin{array}{l}3.05 . \\
12\end{array}$ & $\begin{array}{l}\text { S\&P } \\
\text { (Accordi } \\
\text { ng to } \\
\text { Taiwan } \\
\text { national } \\
\text { Scale) }\end{array}$ & A- & N/A & Negative & N/A & N/A \\
\hline $\begin{array}{l}\text { Hapag } \\
\text { Lloyd }\end{array}$ & $\begin{array}{l}31.10 \\
.12\end{array}$ & Moody's & $\begin{array}{l}\text { B2 } \\
\text { (CFR) } \\
\text { Caa1 } \\
\text { (PDR) }\end{array}$ & $\begin{array}{l}\text { - Market conditions } \\
\text { - Falling demand } \\
\text { - Reducing capacity to } \\
\text { avoid a further } \\
\text { reduction in freight } \\
\text { rates (due to the high } \\
\text { amount of chartered } \\
\text { vessels that could be } \\
\text { redelivered in the next } \\
\text { year) }\end{array}$ & Negative & $\begin{array}{l}\text {-Lower profitability than } \\
\text { rating agency } \\
\text { anticipating previously. }\end{array}$ & $\begin{array}{l}\text { Market } \\
\text { Conditions }\end{array}$ \\
\hline $\begin{array}{l}\text { Mitsui } \\
\text { O.S.K } \\
\text { Lines }\end{array}$ & $\begin{array}{l}8.11 . \\
12\end{array}$ & Moody's & Baа3 & $\begin{array}{l}\text {-Weak earnings and } \\
\text { cash flow } \\
\text {-High financial leverage } \\
\text {-Access to liquidity on } \\
\text { the back of its strong } \\
\text { relationships } \\
\text { Japanese financial } \\
\text { institutions }\end{array}$ & Negative & $\begin{array}{l}\text {-Adverse } \\
\text { environment will continue, } \\
\text { in addition to the } \\
\text { uncertainty in the global } \\
\text { economy }\end{array}$ & Leverage \\
\hline $\begin{array}{l}\text { ZIM } \\
\text { Integrated } \\
\text { Shipping } \\
\text { Services }\end{array}$ & $\begin{array}{l}9.05 . \\
13\end{array}$ & S\&P & CCC & $\begin{array}{l}\text { •Weak liquidity } \\
\text { •Market conditions }\end{array}$ & Negative & $\begin{array}{l}\text {-It is expected that ZIM } \\
\text { restructure its large debt } \\
\text { with banks in near term }\end{array}$ & Liquidity \\
\hline $\begin{array}{l}\text { Nippon } \\
\text { Yusen } \\
\text { Kaisha }\end{array}$ & $\begin{array}{l}22.08 \\
.13\end{array}$ & Moody's & Baa2 & $\begin{array}{l}\text {-Very high debt } \\
\text { leverage } \\
\text {-Oversupply of vessels } \\
\text { in maritime industry } \\
\text { - Low freight rates }\end{array}$ & Negative & $\begin{array}{l}\text {-The ratings could be } \\
\text { downgraded should there be } \\
\text { any indication of a shortfall } \\
\text { in the company's } \\
\text { performance versus } \\
\text { management's publicly } \\
\text { stated guidance for } \\
\text { FY3/2014. }\end{array}$ & Leverage \\
\hline $\begin{array}{l}\text { Mitsui } \\
\text { O.S.K } \\
\text { Lines }\end{array}$ & $\begin{array}{l}22.08 \\
.13\end{array}$ & Moody's & Baa3 & $\begin{array}{l}\text {-Very high debt } \\
\text { leverage } \\
\text {-Market conditions } \\
\text { (oversupply of vessels) }\end{array}$ & Negative & $\begin{array}{l}\text { - Given the negative outlook } \\
\text { and market conditions, an } \\
\text { upgrade is unlikely in the } \\
\text { short-term } \\
\text { - An upgrade will require a } \\
\text { stabilization of the industry } \\
\text { and a significant reduction } \\
\text { in debt leverage }\end{array}$ & Leverage \\
\hline $\begin{array}{l}\text { Hapag } \\
\text { Lloyd }\end{array}$ & $\begin{array}{l}17.09 \\
.13\end{array}$ & Moody's & $\begin{array}{l}\text { B2 } \\
\text { (CFR) } \\
\text { B2 } \\
\text { (PDR) }\end{array}$ & $\begin{array}{l}\text {-Good operating } \\
\text { performance in the first } \\
\text { half of } 2013 \\
\text { •Conditions } \\
\text { container } \\
\text { market } \\
\text {-Decreasing } \\
\text { rates }\end{array}$ & Negative & $\begin{array}{l}\text {-It is expected that the } \\
\text { company to improve its } \\
\text { credit profile by continuing } \\
\text { to reduce costs through its } \\
\text { cost-saving program. } \\
\text { - Bunker costs increasing } \\
\text { beyond } 2013 \text { levels would } \\
\text { put additional pressure on } \\
\text { the ratings }\end{array}$ & $\begin{array}{l}\text { Operating } \\
\text { Performanc } \\
\text { e }\end{array}$ \\
\hline $\begin{array}{l}\text { Hapag } \\
\text { Lloyd }\end{array}$ & $\begin{array}{l}19.09 \\
.13\end{array}$ & Moody’s & Caa1 & $\begin{array}{l}\text { - Hapag Lloyd is a single } \\
\text { entity after the }\end{array}$ & Negative & N/A & $\begin{array}{l}\text { Corporate } \\
\text { Business }\end{array}$ \\
\hline
\end{tabular}




\begin{tabular}{|c|c|c|c|c|c|c|c|}
\hline & & & & $\begin{array}{l}\text { reorganization of Hapag } \\
\text { Lloyd AG Group }\end{array}$ & & & Profile \\
\hline $\begin{array}{l}\text { A.P Moller } \\
\text { Maersk }\end{array}$ & $\begin{array}{l}25.09 \\
.13\end{array}$ & Moody's & Baa1 & $\begin{array}{l}\text {-Company's global } \\
\text { leadership in container } \\
\text { shipping } \\
\text {-Diversified business } \\
\text { profile } \\
\text {-Strong credit metrics, } \\
\text { including moderate } \\
\text { leverage } \\
\text {-A focused and } \\
\text { disciplined } \\
\text { management team } \\
\text {-Solid liquidity } \\
\text {-Market cyclicality }\end{array}$ & Stable & N/A & Size \\
\hline $\begin{array}{l}\text { CMA } \\
\text { CGM }\end{array}$ & $\begin{array}{l}15.10 \\
.13\end{array}$ & Moody’s & B2 & $\begin{array}{l}\text {-More stable operating } \\
\text { and financial profile } \\
\text { following the } \\
\text { completion of its } \\
\text { restructuring } \\
\text { because of ind } \\
\text { strengthened liquidity } \\
\text { position after the sale of } \\
49 \% \text { of its terminal } \\
\text { business and an } \$ 150 \\
\text { million equity injection } \\
\text { as part of its } \\
\text { restructuring }\end{array}$ & Stable & $\begin{array}{l}\text {-CMA CGM will be able to } \\
\text { maintain its current } \\
\text { operating performance, } \\
\text { with the potential for } \\
\text { further improvements over } \\
\text { the next two to three years if } \\
\text { market conditions improve }\end{array}$ & $\begin{array}{l}\text { Operating } \\
\text { Performanc } \\
\text { e }\end{array}$ \\
\hline $\begin{array}{l}\text { Mitsui } \\
\text { O.S.K } \\
\text { Lines }\end{array}$ & $\begin{array}{l}4.08 . \\
14\end{array}$ & Moody's & Review & $\begin{array}{l}\text { - Challenging container } \\
\text { market conditions } \\
\text { effects on earnings } \\
\text { •Expectation of loss } \\
\text { - High leverage for Baa3 } \\
\text { level }\end{array}$ & Negative & $\begin{array}{l}\text {-Possible downgrade } \\
\text {-Increasing investments of } \\
\text { the company }\end{array}$ & $\begin{array}{l}\text { Market } \\
\text { Conditions }\end{array}$ \\
\hline $\begin{array}{l}\text { Mitsui } \\
\text { O.S.K } \\
\text { Lines }\end{array}$ & $\begin{array}{l}4.11 . \\
14\end{array}$ & Moody's & Ваa3 & $\begin{array}{l}\text {-Market conditions (still } \\
\text { oversupply of vessels) } \\
\text { - High debt leverage } \\
\text { - MOL is a core member } \\
\text { of the Sumitomo Mitsui } \\
\text { Group and maintains } \\
\text { firm and stable } \\
\text { relationships with its } \\
\text { main bank } \\
\text { - MOL's strong } \\
\text { relationships with } \\
\text { lenders and group } \\
\text { companies of Japan } \\
\text { support system }\end{array}$ & Negative & $\begin{array}{l}\text {-Moody's expects the } \\
\text { company has turned the } \\
\text { corner and will continue to } \\
\text { de-leverage into next year } \\
\text { - An upgrade is unlikely in } \\
\text { the near or medium term. It } \\
\text { will require sustained profit } \\
\text { and cash flow growth }\end{array}$ & $\begin{array}{l}\text { Market } \\
\text { Conditions }\end{array}$ \\
\hline $\begin{array}{l}\text { Hapag } \\
\text { Lloyd }\end{array}$ & $\begin{array}{l}20.11 \\
.14\end{array}$ & Moody's & Caa1 & $\begin{array}{l}\text { - High cyclicality in the } \\
\text { market } \\
\text { - Hapag-Lloyd was } \\
\text { affected in the past few } \\
\text { years by the combined } \\
\text { effect of low freight } \\
\text { rates, which constrained } \\
\text { the company's } \\
\text { profitability, and large } \\
\text { capital expenditure, } \\
\text { which increased its debt }\end{array}$ & Negative & $\begin{array}{l}\text {-Moody's expects that } \\
\text { Hapag-Lloyd will improve } \\
\text { its credit profile thanks to } \\
\text { its ongoing efforts to boost } \\
\text { its operating efficiency; } \\
\text {-Cost savings derived from } \\
\text { its acquisition of CSAV. } \\
\text { Hapag-Lloyd has estimated } \\
\text { that the merger with CSAV } \\
\text { will result in } \$ 300 \text { million } \\
\text { cost synergies. }\end{array}$ & $\begin{array}{l}\text { Market } \\
\text { Conditions }\end{array}$ \\
\hline
\end{tabular}




\begin{tabular}{|c|c|c|c|c|c|c|c|}
\hline & & & & level. & & & \\
\hline $\begin{array}{l}\text { CMA } \\
\text { CGM }\end{array}$ & $\begin{array}{l}19.12 \\
.14\end{array}$ & Moody’s & B2 & 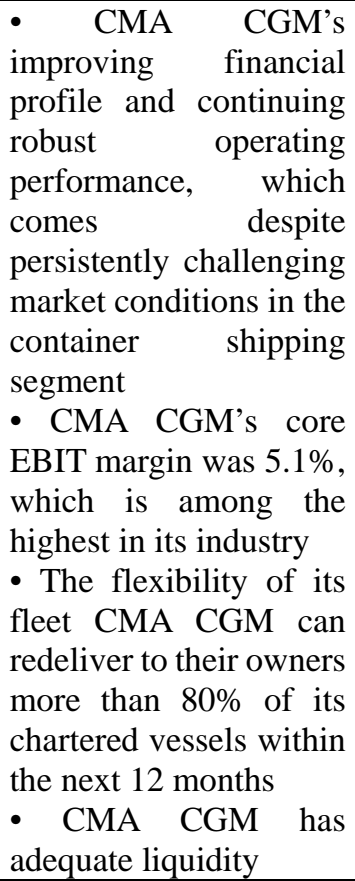 & Positive & $\begin{array}{l}\text {-While freight rates have } \\
\text { remained low and volatile } \\
\text { during 2014, in particular } \\
\text { on the East-West routes, } \\
\text { CMA CGM pursued its } \\
\text { cost-containment efforts } \\
\text { and further reduced its } \\
\text { operating costs per } \\
\text { twenty-foot equivalent unit } \\
\text { (TEU) }\end{array}$ & $\begin{array}{l}\text { Financial } \\
\text { profile }\end{array}$ \\
\hline $\begin{array}{l}\text { Mitsui } \\
\text { O.S.K } \\
\text { Lines }\end{array}$ & $\begin{array}{l}3.02 . \\
15\end{array}$ & Moody’s & Review & $\begin{array}{l}\text {-Much slower earnings } \\
\text { recovery than expected } \\
\text { by Moody's } \\
\text { •MOL's earnings } \\
\text { benefited from the } \\
\text { recent declines in } \\
\text { bunker fuel prices and } \\
\text { the weaker yen } \\
\text {-Loss expectation }\end{array}$ & Negative & •Possible downgrade & $\begin{array}{l}\text { Market } \\
\text { Conditions }\end{array}$ \\
\hline $\begin{array}{l}\text { CMA } \\
\text { CGM }\end{array}$ & $\begin{array}{l}12.05 \\
.15\end{array}$ & Moody’s & B1 & $\begin{array}{l}\text {-Company's continued } \\
\text { robust operating } \\
\text { performance and } \\
\text { resulting improvements } \\
\text { to its financial profile } \\
\text { despite challenging } \\
\text { conditions in the } \\
\text { container market } \\
\text {-Highest profitability } \\
\text { level in the industry }\end{array}$ & Stable & $\begin{array}{l}\text { - These improvements to } \\
\text { persist in } 2015 \text { in spite of } \\
\text { ongoing challenging market } \\
\text { conditions in the container } \\
\text { shipping segment } \\
\text {-CMA CGM will maintain a } \\
\text { top-tier operating } \\
\text { performance and generate } \\
\text { positive free cash flow } \\
\text { within the next 12-18 } \\
\text { months }\end{array}$ & $\begin{array}{l}\text { Market } \\
\text { Conditions }\end{array}$ \\
\hline $\begin{array}{l}\text { Mitsui } \\
\text { O.S.K } \\
\text { Lines }\end{array}$ & $\begin{array}{l}16.06 \\
.15\end{array}$ & Moody’s & Ba1 & $\begin{array}{l}\text {-Expectation that the } \\
\text { company will not be } \\
\text { able to deleverage fast } \\
\text { enough to maintain its } \\
\text { investment grade rating } \\
\text {-Declined ordinary } \\
\text { profit } \\
\text {-Weak earnings and } \\
\text { operating performance } \\
\text {-Difficult market } \\
\text { conditions } \\
\text { - Very high debt } \\
\text { leverage }\end{array}$ & Stable & $\begin{array}{l}\text {-It is expected that the } \\
\text { market will remain weak }\end{array}$ & $\begin{array}{l}\text { Market } \\
\text { Conditions }\end{array}$ \\
\hline $\begin{array}{l}\text { A.P Moller } \\
\text { Maersk }\end{array}$ & $\begin{array}{l}17.06 \\
.15\end{array}$ & Moody’s & Baa1 & $\begin{array}{l}- \text { Market conditions } \\
\text {-Lower oil prices } \\
\text {-Maersk Line's }\end{array}$ & Positive & $\begin{array}{l}\text {-It is expected APMM's } \\
\text { leverage ratio to increase in } \\
2015 \text { to close to } 2 x\end{array}$ & $\begin{array}{l}\text { Market } \\
\text { Conditions }\end{array}$ \\
\hline
\end{tabular}




\section{Ml Macrothink}

Business and Management Horizons

ISSN 2326-0297

2017, Vol. 5, No. 2

\begin{tabular}{|c|c|c|c|c|c|c|c|}
\hline & & & & $\begin{array}{l}\text { continuing cost-cutting } \\
\text { efforts }\end{array}$ & & & \\
\hline $\begin{array}{l}\text { CMA } \\
\text { CGM }\end{array}$ & $\begin{array}{l}17.06 \\
.15\end{array}$ & Moody’s & B1 & •Profitability level & Positive & $\begin{array}{l}\text { - These improvements to } \\
\text { persist in } 2015 \text { in spite of } \\
\text { ongoing challenging market } \\
\text { conditions in the container } \\
\text { shipping segment }\end{array}$ & $\begin{array}{l}\text { Profitabilit } \\
\text { y }\end{array}$ \\
\hline $\begin{array}{l}\text { Hapag } \\
\text { Lloyd }\end{array}$ & $\begin{array}{l}17.06 \\
.15\end{array}$ & Moody's & B2 & $\begin{array}{l}\text { - Reduction in the debt } \\
\text { adjustment related to } \\
\text { operating leases } \\
\text {-Its increased scale from } \\
\text { the integration of the } \\
\text { recently-acquired } \\
\text { container shipping } \\
\text { activities of the Chilean } \\
\text { company Compania } \\
\text { Sud Americana de } \\
\text { Vapores (CSAV, } \\
\text { unrated); }\end{array}$ & Stable & $\begin{array}{l}\text {-It is expected that } \\
\text { Hapag-Lloyd will improve } \\
\text { its credit profile thanks to } \\
\text { its ongoing efforts to boost } \\
\text { its operating efficiency } \\
\text { - Cost savings derived from } \\
\text { the integration of CSAV } \\
\text { (estimated at } \$ 300 \text { million). }\end{array}$ & $\begin{array}{l}\text { Operating } \\
\text { Performanc } \\
\text { e }\end{array}$ \\
\hline $\begin{array}{l}\text { Nippon } \\
\text { Yusen } \\
\text { Kaisha }\end{array}$ & $\begin{array}{l}17.06 \\
.15\end{array}$ & Moody's & Baa2 & $\begin{array}{l}\cdot \text {-NYK's position as one } \\
\text { of the world's largest } \\
\text { shipping companies } \\
\text {-NYK's ordinary profit } \\
\text { surged } 44 \% \text { to JPY } 84.0 \\
\text { billion from JPY } 58.4 \\
\text { billion a year ago } \\
\text { - Reduction of costs due } \\
\text { to low oil prices } \\
\text { supports earnings }\end{array}$ & Stable & $\begin{array}{l}\text {-Moody's experts expect } \\
\text { that modest earnings } \\
\text { growth and debt reduction, } \\
\text { which together will lead to } \\
\text { continuous and gradual } \\
\text { deleveraging }\end{array}$ & Size \\
\hline $\begin{array}{l}\text { Hapag } \\
\text { Lloyd }\end{array}$ & $\begin{array}{l}29.09 \\
.15\end{array}$ & Moody’s & B2 & $\begin{array}{l}\text { - Company's improved } \\
\text { operating performance } \\
\text { since the beginning of } \\
2015 \text {, driven by the } \\
\text { lower bunker fuel price } \\
\text { and fundamental } \\
\text { changes to its } \\
\text { operations and cost } \\
\text { structure. } \\
\text {-During H1 2015, } \\
\text { Hapag-Lloyd reported } \\
\text { EBITDA of EUR493 } \\
\text { million, a significant } \\
\text { increase from EUR67 } \\
\text { million in H1 2014. }\end{array}$ & Positive & $\begin{array}{l}\text {-Moody's expects that these } \\
\text { improvements will further } \\
\text { improve Hapag-Lloyd's } \\
\text { financial profile in the } \\
\text { coming quarters, despite } \\
\text { challenging market } \\
\text { conditions in the container } \\
\text { shipping industry. }\end{array}$ & $\begin{array}{l}\text { Operating } \\
\text { Performanc } \\
\text { e }\end{array}$ \\
\hline $\begin{array}{l}\text { CMA } \\
\text { CGM }\end{array}$ & $\begin{array}{l}7.12 . \\
15\end{array}$ & Moody’s & B1 & $\begin{array}{l}\text {-CMA CGM's potential } \\
\text { acquisition of NOL } \\
\text { would strengthen its } \\
\text { business profile } \\
\text { •CMA CGM's capacity } \\
\text { by approximately a } \\
\text { third (based on pro } \\
\text { forma September } 2015 \\
\text { data), consolidating its } \\
\text { position as the } \\
\text { third-largest player in } \\
\text { the container shipping } \\
\text { segment }\end{array}$ & Stable & $\begin{array}{l}\text {-CMA CGM's financial } \\
\text { profile will return to a } \\
\text { profile in line with the B1 } \\
\text { rating within } 18 \text { months } \\
\text { after the closing of the NOL } \\
\text { acquisition } \\
\text {-CMA CGM will maintain } \\
\text { an adequate liquidity profile } \\
\text { and refinance the } \\
\text { acquisition financing well } \\
\text { in advance of its maturity }\end{array}$ & $\begin{array}{l}\text { Corporate } \\
\text { Business } \\
\text { Profile }\end{array}$ \\
\hline $\begin{array}{l}\text { Mitsui } \\
\text { O.S.K }\end{array}$ & $\begin{array}{l}4.02 . \\
16\end{array}$ & Moody’s & Ba1 & $\begin{array}{l}\text {-Weaker than expected } \\
\text { profitability for the }\end{array}$ & Negative & $\begin{array}{l}\text {-It is expected that the } \\
\text { container segment will }\end{array}$ & $\begin{array}{l}\text { Profitabilit } \\
\mathrm{y}\end{array}$ \\
\hline
\end{tabular}




\section{Macrothink Mnstitute}

\begin{tabular}{|c|c|c|c|c|c|c|c|}
\hline Lines & & & & $\begin{array}{l}\text { fiscal year due to the } \\
\text { challenging market } \\
\text { conditions } \\
\text { - Volatility of the } \\
\text { operating environment } \\
\text { - Weak level of earnings } \\
\text { - Higher leverage than } \\
\text { expected }\end{array}$ & & $\begin{array}{l}\text { remain in oversupply for at } \\
\text { least the next } 12-18 \text { months, } \\
\text { potentially depressing } \\
\text { pricing further }\end{array}$ & \\
\hline $\begin{array}{l}\text { A.P Moller } \\
\text { Maersk }\end{array}$ & $\begin{array}{l}16.02 \\
.16\end{array}$ & Moody's & Baa1 & $\begin{array}{l}\text { - Challenging market } \\
\text { conditions (oversupply) } \\
\text {-Sharply decline of } \\
\text { freight rates } \\
\text {-EBITDA down by } 21 \%\end{array}$ & Stable & $\begin{array}{l}\text {-Cash flow generation from } \\
\text { operations, which Moody's } \\
\text { expects to be } \$ 5 \text { billion- } \$ 6 \\
\text { billion in the next } 12 \\
\text { months }\end{array}$ & $\begin{array}{l}\text { Market } \\
\text { Conditions }\end{array}$ \\
\hline $\begin{array}{l}\text { Mitsui } \\
\text { O.S.K } \\
\text { Lines }\end{array}$ & $\begin{array}{l}6.05 \\
16\end{array}$ & Moody's & Review & $\begin{array}{l}- \text { Low freight rates } \\
\cdot \text { Expected earnings } \\
\text { decline }\end{array}$ & Negative & -Possible downgrade & $\begin{array}{l}\text { Market } \\
\text { Conditions }\end{array}$ \\
\hline $\begin{array}{l}\text { Nippon } \\
\text { Yusen } \\
\text { Kaisha }\end{array}$ & $\begin{array}{l}10.05 \\
.16\end{array}$ & Moody’s & Baa2 & $\begin{array}{l}\text {-High debt leverage } \\
\text { •Oversupply of vessels } \\
\text { in maritime industry } \\
\text { - Low freight rates }\end{array}$ & Negative & $\begin{array}{l}\text {-An upgrade is unlikely in } \\
\text { the near term }\end{array}$ & Leverage \\
\hline $\begin{array}{l}\text { Nippon } \\
\text { Yusen } \\
\text { Kaisha }\end{array}$ & $\begin{array}{l}3.08 . \\
16\end{array}$ & Moody’s & Review & $\begin{array}{l}\text { •Slower earnings } \\
\text { recovery than expected }\end{array}$ & Negative & -Possible downgrade & $\begin{array}{l}\text { Profitabilit } \\
\text { y }\end{array}$ \\
\hline $\begin{array}{l}\text { Mitsui } \\
\text { O.S.K } \\
\text { Lines }\end{array}$ & $\begin{array}{l}3.8 .1 \\
6\end{array}$ & Moody’s & Ba1 & $\begin{array}{l}\text { - Realized half of the } \\
\text { expected earnings } \\
\text { - Low freight rates due } \\
\text { to oversupply of vessels } \\
\text {-Very high debt } \\
\text { leverage }\end{array}$ & Negative & $\begin{array}{l}\text {-An upgrade for MOL is } \\
\text { unlikely in the near term } \\
\text { given the current } \\
\text { challenging } \\
\text { conditions }\end{array}$ & $\begin{array}{l}\text { Profitabilit } \\
\text { y }\end{array}$ \\
\hline $\begin{array}{l}\text { Nippon } \\
\text { Yusen } \\
\text { Kaisha }\end{array}$ & $\begin{array}{l}2.11 . \\
16\end{array}$ & Moody’s & Ваa3 & $\begin{array}{l}\text {-Low cash flow due to } \\
\text { low freight rates } \\
\text {-Slower financial } \\
\text { recovery than expected }\end{array}$ & Negative & $\begin{array}{l}\text {-Moody’s expects ordinary } \\
\text { loss end of the financial } \\
\text { year }\end{array}$ & $\begin{array}{l}\text { Market } \\
\text { Conditions }\end{array}$ \\
\hline $\begin{array}{l}\text { Hyundai } \\
\text { Merchant } \\
\text { Marine }\end{array}$ & $\begin{array}{l}13.02 \\
.17\end{array}$ & Moody’s & $\begin{array}{l}\text { BB } \\
\text { (from } \\
\text { D) }\end{array}$ & $\begin{array}{l}\text {-After bankruptcy of } \\
\text { Hanjin, HMM gained } \\
\text { good share of Hanjin's } \\
\text { customer } \\
\text {-Increasing market } \\
\text { share }\end{array}$ & N/A & N/A & Size \\
\hline
\end{tabular}

As seen in Table 4, market conditions are the factor that most affect maritime company credit rating actions. In 23 of the 69 observations, market conditions are disclosed as the main factor; and in 15 observations, the effect is negative. Contrarily, when the external market conditions improve, rating actions were affected positively. 
Table 4. Relevant factors in determining maritime company credit rating actions

\begin{tabular}{|l|l|l|l|l|l|}
\hline Main Determinant & Negative & Positive & Stable & N/A & Grand Total \\
\hline Market Conditions & 15 & 2 & 5 & 1 & 23 \\
\hline Operating Performance & 1 & 3 & 6 & & 10 \\
\hline Leverage & 6 & 1 & 2 & & 9 \\
\hline Profitability & 3 & 2 & 2 & & 7 \\
\hline Financial profile & & 2 & 3 & & 5 \\
\hline N/A & 4 & & & 1 & 5 \\
\hline Size & & & 3 & 1 & 4 \\
\hline Corporate Business Profile & 1 & 1 & 1 & & 3 \\
\hline Cash flow generation & & & 1 & & 1 \\
\hline Growth & & 1 & & & 1 \\
\hline Liquidity & 1 & & & & 1 \\
\hline Grand Total & $\mathbf{3 1}$ & $\mathbf{1 2}$ & $\mathbf{2 3}$ & $\mathbf{3}$ & $\mathbf{6 9}$ \\
\hline
\end{tabular}

The second most effective factor is operating performance. The disclosures on operating performance mostly lead to stable credit ratings. While the third most effective factor, high leverage is mostly associated with negative credit rating actions. Other effective company-specific factors are profitability, financial profile, company size, corporate business profile, cash flow generation, company growth and liquidity. The "financial profile" and "corporate business profile" factors are more opaque than other factors. Disclosures on financial profile generally states an improvement in the financial profile, yet does not indicate the company-specific financial elements. Similarly, corporate business profile is mostly linked to the company and its' subsidiaries characteristics, however, the disclosures lack detailed information on these characteristics.

When the observations are examined by years, as shown in Table 5, it is seen that the macro-economic factor, market conditions, are dominant in determining the credit rating actions mostly in 2000, 2001, and after 2008.

Overall, the results indicate that when there is a global economic downturn, company-specific factors are less effective in determining maritime company credit ratings.

Table 5. Relevant factors by years

\begin{tabular}{|l|l|l|l|l|l|}
\hline Year/ Relevant Factor & N/A & Negative & Positive & Stable & Grand Total \\
\hline 2000 & & & 2 & & 2 \\
\hline Corporate Business Profile & & & 1 & & 1 \\
\hline Market Conditions & & & 1 & & 1 \\
\hline 2001 & & & & 2 & 2 \\
\hline Market Conditions & & & & 2 & 2 \\
\hline 2002 & & & & 1 & 1 \\
\hline
\end{tabular}




\begin{tabular}{|c|c|c|c|c|c|}
\hline Leverage & & & & 1 & 1 \\
\hline 2003 & & & & 1 & 1 \\
\hline Leverage & & & & 1 & 1 \\
\hline 2004 & & & & 4 & 4 \\
\hline Cash flow generation & & & & 1 & 1 \\
\hline Operating Performance & & & & 3 & 3 \\
\hline 2005 & & & 3 & 2 & 5 \\
\hline Financial profile & & & & 1 & 1 \\
\hline Growth & & & 1 & & 1 \\
\hline Operating Performance & & & 1 & & 1 \\
\hline Profitability & & & 1 & 1 & 2 \\
\hline 2006 & & & & 1 & 1 \\
\hline Size & & & & 1 & 1 \\
\hline 2007 & & & 2 & 1 & 3 \\
\hline Financial profile & & & 1 & & 1 \\
\hline Leverage & & & 1 & & 1 \\
\hline Profitability & & & & 1 & 1 \\
\hline 2008 & & 2 & & & 2 \\
\hline Market Conditions & & 1 & & & 1 \\
\hline N/A & & 1 & & & 1 \\
\hline 2009 & 1 & 4 & & & 5 \\
\hline Leverage & & 1 & & & 1 \\
\hline Market Conditions & & 3 & & & 3 \\
\hline N/A & 1 & & & & 1 \\
\hline 2010 & & 1 & & 2 & 3 \\
\hline Financial profile & & & & 1 & 1 \\
\hline Leverage & & 1 & & & 1 \\
\hline Operating Performance & & & & 1 & 1 \\
\hline 2011 & & 3 & & 1 & 4 \\
\hline Financial profile & & & & 1 & 1 \\
\hline Market Conditions & & 2 & & & 2 \\
\hline N/A & & 1 & & & 1 \\
\hline 2012 & 1 & 6 & 1 & & 8 \\
\hline Leverage & & 1 & & & 1 \\
\hline Market Conditions & 1 & 3 & & & 4 \\
\hline N/A & & 2 & & & 2 \\
\hline Operating Performance & & & 1 & & 1 \\
\hline 2013 & & 5 & & 2 & 7 \\
\hline Corporate Business Profile & & 1 & & & 1 \\
\hline Leverage & & 2 & & & 2 \\
\hline Liquidity & & 1 & & & 1 \\
\hline Operating Performance & & 1 & & 1 & 2 \\
\hline
\end{tabular}




\begin{tabular}{|l|l|l|l|l|l|}
\hline Size & & & & 1 & 1 \\
\hline 2014 & & 3 & 1 & & 4 \\
\hline Financial profile & & & 1 & & 1 \\
\hline Market Conditions & & 3 & & & 3 \\
\hline 2015 & & 1 & 3 & 5 & 9 \\
\hline Corporate Business Profile & & & & 1 & 1 \\
\hline Market Conditions & & 1 & 1 & 2 & 4 \\
\hline Operating Performance & & & 1 & 1 & 2 \\
\hline Profitability & & & 1 & & 1 \\
\hline Size & & & & 1 & 1 \\
\hline 2016 & & 6 & & 1 & 7 \\
\hline Leverage & & 1 & & & 1 \\
\hline Market Conditions & & 2 & & 1 & 3 \\
\hline Profitability & & 3 & & & 3 \\
\hline 2017 & 1 & & & & 1 \\
\hline Size & 1 & & & & 1 \\
\hline Grand Total & $\mathbf{3}$ & $\mathbf{3 1}$ & $\mathbf{1 2}$ & $\mathbf{2 3}$ & $\mathbf{6 9}$ \\
\hline
\end{tabular}

\section{Conclusion}

Maritime demand is driven by global economic growth and transportation needs. Since the 2008 financial crisis, world trade volume has aggressively declined. In turn, we see that the maritime industry was one of the most negatively affected industries by the crisis (UNCTAD, 2010). Maritime companies are also exposed to natural disasters and have been threatened by political unrest. These factors, coupled with rising and volatile energy and commodity prices have negatively affected the industry. With an increase in demand in 2010, a positive turnaround in trade volume was recorded, particularly in the container trade. However, the future outlook and the over-supply issues remain critical since the sector is vulnerable on the same indefiniteness and shocks that face the world economy (UNCTAD, 2011).

Our findings show that macro-economic factors determine the credit ratings of maritime companies. Specifically, after the 2008 financial crisis, the CRA disclosures on company specific factors have dramatically decreased.

The reason behind this finding may be the opaque nature of maritime companies and high costs of obtaining private information to evaluate firm-specific factors. However, opacity prevails in credit rating actions in line with the arguments of Bona \& Ribeiro (2009), Elkhoury (2008) and Murcia et al. (2014). Although the disclosures on external market conditions provides insight, in most observations, factors such as "financial profile" and "corporate business profile" lack detailed information disclosed to the investors. Thus, it may be concluded that the credit rating process of maritime companies lacks transparency, and rely mostly on market risks.

The main limitation of this study is the sample size. An examination into the reasons behind 
the issue of opaqueness, and the ways to enhance the disclosed information and transparency for maritime company credit rating actions, could be considered as future research.

\section{References}

A. P. Moller Maersk. (2016). Annual Report. [Online] Available: https://www.maersk.com/press/press-release-archive/annual-report-2016

Ackermann, J. (2008). The subprime crisis and its consequences. Journal of Financial Stability, 4(4), 329-337. https://doi.org/10.1016/j.jfs.2008.09.002

Altman, E. I. (1968). Financial ratios, discriminant analysis and the prediction of corporate bankruptcy. The Journal of Finance, 23(4), 589-609. http://dx.doi.org/10.1111/j.1540-6261.1968.tb00843.x

Atiya, A. F. (2001). Bankruptcy prediction for credit risk using neural networks: A survey and new results. IEEE Transactions on Neural Networks, 12(4), 929-935. https://doi.org/10.1109/72.935101

Beaver, W. (1966). Financial ratios as predictors of failure. Journal of Accounting Research, 4(Empirical research in accounting: selected studies), 71-111. https://doi.org/10.2307/2490171

Bone, R. B., \& Ribeiro, E. P. (2009). Conteúdo informacional dos ratings corporativos de empresas brasileiras, 1995-2007. Anais do Encontro Brasileiro de Finanças, São Leopoldo, RS, Brasil, 9.

Brauner, A. (1994). Shipping finance: A current view of the availability of finance and financiers' requirements for vessel quality. International Conference on Maritime Safety, Istanbul: Chamber of Shipping Publications, 109-143.

Cantor, R., \& Packer, F. (1994). The Credit Rating Industry. Federal Reserve Bank of New York Quarterly Review, Summer/Fall, 1-26.

CMA CGM. (2016) Annual Report. [Online] Available: https://www.cma-cgm.com/static/Finance/PDFFinancialRelease/FY\%202016\%20-\%20Annu al\%20Consolidated\%20Accounts.pdf

COSCO. (2016), Annual Report. [Online] Available: https://www.wertpapier-forum.de/applications/core/interface/file/attachment.php?id=106702

De Monie, G., Rodrigue, J. P., \& Notteboom, T. (2009). Economic cycles in maritime shipping and ports: The path to the crisis of 2008. In P. V. Hall, R. J. McCalla, C. Comtois \& B. Slack (Eds.), Integrating Seaports and Trade Corridors (pp. 13-30, Aldershot: Ashgate).

Dichev, I. D. (1998). Is the risk of bankruptcy a systematic risk? The Journal of Finance, 53(3), 1131-1147. http://dx.doi.org/10.1111/0022-1082.00046

Elkhoury, M. (2008). Credit rating agencies and their potential impact on developing countries. United Nations Conference on Trade and Development Discussion Papers, No.186, 
Geneva.

Evergreen. (2016). Financial Reports [Online] Available: https://www.evergreen-marine.com/tbf1/pdf/2016AR.pdf

Fitzpatrick, P. (1932). A Comparison of the Ratios of Successful Industrial Enterprises with Those of Failed Companies. The Accountants Publishing Company.

Gonzales, F., Haas, F., Ohannes, R., Persson, J. M., Toledo, L., Violi, R., Zins, C., \& Wieland, M. (2004). Market dynamics associated with credit ratings: a literature review. Banque de France Financial Stability Review, 4, 53-76.

Hamburg Süd. (2016). Fact \& Figures. [Online] Available: https://www.hamburgsud.com/group/en/corporatehome/company/facts_figures/index.html

Hanjin Financial Data. (2017). [Online] Available: https://www.investing.com/equities/hanjin-shippin-balance-sheet

Hapag Lloyd. (2016). Annual Report. [Online] Available: https://www.hapag-lloyd.com/content/dam/website/downloads/pdf/HLAG_Group_Annual_R eport_2016.pdf

Hyundai Merchant Marine. (2016). Annual Report. [Online] Available: http://engkind.krx.co.kr/disclosure/searchdisclosurebycorp.do?method=searchDisclosureByC orpMain\&searchCodeType=char\&searchCorpName=HYUNDAI\%20MERC\%20MAR\&lang $\mathrm{TpCd}=1$

Kawasaki Lines. (2016). Financial Report. [Online] Available: http://www.kline.co.jp/en/ir/library/annual/_icsFiles/afieldfile/2016/09/21/KlineReport2016 _en.pdf

Kisgen, D. J., \& Strahan, P. E. (2010). Do Regulations Based on Credit Ratings Affect a Firm's Cost of Capital? The Review of Financial Studies, 23(12), 4324-4347. https://doi.org/10.1093/rfs/hhq077

Lo Re, M. L. (2011). Impact of the Global Financial Crisis on the EU and Euro Area vs. the USA. China-USA Business Review, 10(7), 532-538.

Matthies, A. B. (2013). Empirical Research on Corporate Credit- Ratings: A Literature Review. SFB 649 Discussion Paper 2013-003.

Mayer-Brown. (2014). The restructuring of non-performing shipping loans: Learning from experience in the real estate crisis. Legal Update. [Online] Available: https://www.mayerbrown.com/files/Publication/baaefb92-d2d9-4dc2-b248-26ad2556cfab/Pre sentation/PublicationAttachment/1854f2fd-307c-411e-98ab-286d8f780488/140226-FRK-Shi p-Loans.pdf

Melvin, M., \& Taylor, M. P. (2009). The global financial crisis: Causes, threats and opportunities. Introduction and overview. Journal of International Money and Finance, 28(8), 1243-1245. https://doi.org/10.1016/j.jimonfin.2009.08.002 
Mitsui Osaka Lines. (2016). Annual Report. [Online] Available: http://www.mol.co.jp/en/ir/data/annual/ar-e2016s.html

MSC. (2016). Financial Report. [Online] Available: https://www.msc.com/grc/about-us/financial-results?lang=ko-kr

Murcia, F. C. de S., Murcia, F. D., Rover, S., \& Borba, J. A. (2014). The Determinants of Credit Rating: Brazilian Evidence. BAR- Brezilian Administration Review, 11(2), 188-209. http://dx.doi.org/10.1590/S1807-76922014000200005

Neptune Orient Lines. (2016). Facts on Neptune Orient Lines. [Online] Available: https://www.cma-cgm.com/static/Finance/PDFFinancialRelease/FY\%202016\%20-\%20Annu al\%20Consolidated\%20Accounts.pdf

Nippon Yusen Kaisha. (2016). Financial Data and Shipping Market Data Factbook II. [Online] Available: http://www.nyk.com/english/ir/library/fact02/pdf/2016_factbook02_04.pdf

Odom, M., \& Sharda, R. (1990). A neural network model for bankruptcy prediction. IJCNN International Joint Conference on Neural Networks. 17-21 June, San Diego, CA, USA. 2: 163-168. https://doi.org/10.1109/IJCNN.1990.137710

Ong, M. K. (2003). Credit Ratings Methodologies, Rationale and Default Risk. London: Risk Waters Group.

OOCL. (2016). Annual Report. [Online] Available: https://www.ooilgroup.com/financials/interimandannualreports/2016/Documents/E-00316AR -10032017.pdf

Pacific International Lines. (2016). Financial Report. [Online] Available: https:/www.pilship.com/PIL\%201H\%202017\%20FS\%20(Final).pdf

Rajendran, M. M. A. (2015). Credit Risk Management and Insurance Practices-An Overview. Journal of Research in Business, Economics and Management, 2(2), 89-96.

Ramser, J., \& Foster, L. (1931). A Demonstration of Ratio Analysis. Bulletin 40. University of Illinois, Bureau of Business Research, Urbana, IL

Saunders, A., Allen, L., \& Boudoukh, J. (2004). Understanding Market, Credit, and Operational Risk: The Value of Risk Approach. London: Blackwell Publishing.

Standard \& Poor's, Rating Criteria and Models. (2017). [Online] Available: https://www.standardandpoors.com/en_US/web/guest/ratings/ratings-criteria

Stopford, M. (2013). The financial crisis: Its impact on shipping. ICS Symposium, British Library. [Online] Available: http://www.ics-shipping.org/docs/default-source/ics-conference-2013/martin-stopford---the-fi nancial-crisis.pdf?sfvrsn=2

UASC. (2017). [Online] Available: http://www.uasc.net/en/company-profile

UNCTAD Review of Maritime Transport. (2010). United Nations Conference on Trade and 


\section{Macrothink}

Business and Management Horizons

ISSN 2326-0297

2017, Vol. 5, No. 2

Development. [Online] Available: http://unctad.org/en/docs/rmt2010_en.pdf

UNCTAD Review of Maritime Transport. (2011). United Nations Conference on Trade and Development. [Online] Available: http://unctad.org/en/docs/rmt2011_en.pdf

UNCTAD Review of Maritime Transport. (2016). United Nations Conference on Trade and Development. [Online] Available: http://unctad.org/en/PublicationsLibrary/rmt2016_en.pdf

Wan Hai Lines Ltd. (2016). Annual Report. [Online] Available: http://www.wanhai.com/views/content/ContentList.xhtml?file_num=67057\&parent_id=6706 5\&top_file_num=65578

Wilson, R. L., \& Sharda, R. (1994). Bankruptcy prediction using neural networks. Decision Support Systems, 11(5), 545-557 https://doi.org/10.1016/0167-9236(94)90024-8

Winakor, A., \& Smith, R. (1935). Changes in the Financial Structure of Unsuccessful Industrial Corporations. Bulletin 51. Bureau of Business Research, University of Illinois, Urbana, IL

X-Press Feeder. (2017). [Online] Available: https://www.X-pressfeeders.com/Home/About

Yang, M. (2016). Financial Report. [Online] Available: https://www.yangming.com/files/Investor_Relations/2016(Consolidated).pdf

ZIM. (2016). Integrated Shipping Operating and Financial Review. [Online] Available: http://www.zim.com/aboutus/sr/operating\%20and\%20financial\%20review\%20for\%20the\%2 0year\%20ended\%20december\%2031\%202016.pdf

\section{Copyright Disclaimer}

Copyright for this article is retained by the author(s), with first publication rights granted to the journal.

This is an open-access article distributed under the terms and conditions of the Creative Commons Attribution license (http://creativecommons.org/licenses/by/3.0/). 